\title{
Special Issue on: Twelfth International Conference on Ontologies, Databases, and Applications of Semantics
}

\author{
Dejing Dou ${ }^{1}$ - Pieter De Leenheer ${ }^{2}$
}

Published online: 15 May 2015

(C) Springer-Verlag Berlin Heidelberg 2015

To foster the dissemination of the best ideas and results, the Journal on Data Semantics (JoDS) has a tradition to publish extended versions of the best papers from selected conferences whose scope encompasses or intersects the scope of the journal.

This issue includes the extended versions of four selected best papers from the Twelfth International Conference on Ontologies, Databases, and Applications of Semantics (ODBASE 2013), which took place in September, 2013 in Graz, Austria.

Originally, five papers from ODBASE 2013 were selected based on their quality, relevance and significance, and the viability of extending their results. All extended papers went through a strict peer review process, and the authors were required to revise the papers based on the concerns expressed by the reviewers. Finally, four papers were accepted and one was rejected.

The first paper, by Lukasiewicz et al., introduces two models for "Preference-Based Query Answering in Probabilistic Datalog+/- Ontologies:" one representing user preferences and one representing the (probabilistic) uncertainty with which inferences are made. The paper proposes four specific operators and studies their semantic and computational properties. The paper also provides an algorithm for ranking answers based on the iteration of the well-known skyline answers to a query and shows that, under certain conditions, it runs in polynomial time in the data complexity.

In collaborative and interoperable information systems it is frequently necessary to export a subset of an ontology, in

\footnotetext{
$凶$ Dejing Dou

dou@cs.uoregon.edu

1 University of Oregon, Eugene, USA

2 Collibra, USA
}

particular to export all instances and axioms subsumed by a shared domain ontology. The second paper, by Köpke et al., proposes methods for "Efficient Projection of Ontologies." These methods are based on a deep analysis of properties of axiom sets that can be used to efficiently filter redundancies during the export process and provide methods to further minimize redundancies in the result. The paper evaluates the performance of the approach using standard benchmarking ontologies with large Aboxes and a use case of the biomedical domain.

The third paper, by Wang et al., introduces methods for "Analysis and Prediction of User Editing Patterns in Ontology Development Projects." The paper uses data mining technique, specifically the association rule mining, to investigate how to predict the next editing operation that a user will make based on the change history of two ontologies, ICTM and ICD-11, which have been developed in WebProtégé, a web-based open-source ontology editing environment. The paper evaluates the impact of different training and testing window sizes on the prediction accuracies. The paper also evaluates prediction accuracies across different user groups and different ontologies.

The fourth paper, by Geibel et al., introduces "OntologyBased Information Extraction: Identifying Eligible Patients for Clinical Trials in Neurology." The designed clinical research data warehouse makes use of information extracted from clinical documents like admission reports, radiological findings, and discharge letters. It is supported by clinical application ontologies, which enable the identification of main terms and their properties, as well as semantic search with synonyms, hypernyms, and syntactic variants. The paper provides a thorough evaluation of the deployed systems based on real data related to clinical trials conducted by the neurology departments.

Guest Editors 